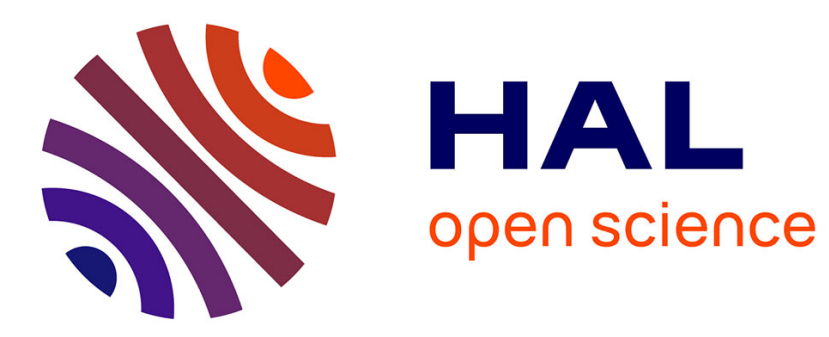

\title{
Correlated Disordered Plasmonic Nanostructures Arrays for Augmented Reality
}

Hervé Bertin, Yoann Brûlé, Giovanni Magno, Thomas Lopez, Philippe Gogol, Laetitia Pradere, Boris Gralak, David Barat, Guillaume Demésy, Béatrice Dagens

\section{To cite this version:}

Hervé Bertin, Yoann Brûlé, Giovanni Magno, Thomas Lopez, Philippe Gogol, et al.. Correlated Disordered Plasmonic Nanostructures Arrays for Augmented Reality. ACS photonics, 2018, 5 (7), pp.2661 - 2668. 10.1021/acsphotonics.8b00168 . hal-01907011

\section{HAL Id: hal-01907011 https://hal.science/hal-01907011}

Submitted on 30 Aug 2021

HAL is a multi-disciplinary open access archive for the deposit and dissemination of scientific research documents, whether they are published or not. The documents may come from teaching and research institutions in France or abroad, or from public or private research centers.
L'archive ouverte pluridisciplinaire HAL, est destinée au dépôt et à la diffusion de documents scientifiques de niveau recherche, publiés ou non, émanant des établissements d'enseignement et de recherche français ou étrangers, des laboratoires publics ou privés. 


\title{
Correlated disordered plasmonic nanostructures
}

\section{arrays for augmented reality}

\author{
${ }^{1}$ Herve Bertin, ${ }^{2}$ Yoann Brûlé, ${ }^{1}$ Giovanni Magno, ${ }^{1}$ Thomas Lopez, ${ }^{1}$ Philippe Gogol, ${ }^{3}$ Laetitia \\ Pradere, ${ }^{2}$ Boris Gralak, ${ }^{3}$ David Barat, ${ }^{2}$ Guillaume Demésy, ${ }^{1}$ Beatrice Dagens $*$
}
${ }^{1}$ Centre de Nanosciences et de Nanotechnologies, CNRS, Université Paris-Sud, Université Paris- Saclay, C2N-Orsay, 91405 Orsay Cedex, France
${ }^{2}$ Aix Marseille Univ, CNRS, Centrale Marseille, Institut Fresnel, Marseille, France
${ }^{3}$ PSA groupe, Direction Scientifique, Centre technique de Vélizy, route de Gisy, Vélizy- Villacoublay, F-78140, France

Author e-mail address: beatrice.dagens@,c2n.upsaclay.fr

KEYWORDS. Plasmonics, Wavelength filtering devices, Nanostructure fabrication, Metallic nanoparticles, Metasurface, Visualization, Localized surface plasmon, Lattice surface mode.

\begin{abstract}
Plasmonic resonators are excellent candidates to control reflectance of functionalized substrates. Because of their subwavelength characteristic dimensions, they can even be used to modify the color of transparent glass plates without altering the transparency quality. Their spatial arrangement must be carefully chosen so that the plates don't produce nonspecular diffraction, whatever their spatial density. We compare here the response of silver
\end{abstract}


nanoparticles (NPs) arrays with different NPs sizes, spatial densities, and arrangements (periodic and correlated disordered). The effects of these geometrical parameters are analyzed in detail by measuring the reflectance and transmittance spectra in visible wavelength. We show that correlated disordered gratings attenuate diffraction effects appearing at lower spatial densities while keeping similar reflectance and transmittance responses and maintaining clear transparency of the glass plate. Promising configurations for head-up displays and applications in augmented reality emerge from this study.

Augmented reality is a fast-growing concept which brings many functionalities enhancing efficiency and making life easier for users. This concept can now be addressed thanks to the smart combination of new technologies and can be applied in a wide range of societal issues like education, industry, and medicine. Head-up displays (HUD), already used in the aviation and the automotive domains, can be seen as one early development of augmented reality systems. The principle of HUD consists in reflecting an image generated by a colored display on a glass screen in the driver's field of view. In this configuration, the driver reads 'at a glance' relevant information in a virtual image created at a sufficient distance in front of him to avoid visual accommodation. At the same time, the driver must be able to see the road or the landscape in front of the vehicle. The whole optical system should thus include lenses to collimate and magnify the projected information and also a highly transparent semi-reflective plate. The latter should simultaneously provide significant angular tolerance, efficiently reflect the colors of the display (so that the information remains readable even in case of a sunny weather), provide a clear aberration-free reflected picture and preserve the visual quality of the road through the plate. In the long run, this optical system should be as compact as possible and at the same time wide enough to be inserted in the windshield. The most common technology used to modify the 
spectral reflectance of a wide glass surface is multilayered coatings. Nevertheless, such a technology is limited by the almost uniformity of the coatings and its manufacturing cost remains high. More sophisticated structures based on dielectric resonant gratings (1) also provide enhanced reflectance at a given wavelength, but their low angular tolerance is incompatible with HUD systems.

During the last decade, metallic nanoparticles (NP) resonances, like LSPR (localized surface plasmon resonance) or nanoholes array EOT (extraordinary optical transmission), have received a keen interest for their ability not only to concentrate light at the nanoscale but also to modify the apparent color of wafers. Thanks to the nanoscale structuration, the surface optical properties like the reflectance or the apparent color can be locally controlled with a high spatial resolution down to subwavelength dimensions. Exploiting the EOT mechanism (2), nanohole arrays in metal offer new possibilities for the filtering application. The typical device is made of a subwavelength patterned grating in a perforated metal film (3) and is back-lighted for color filtering (4) or for printing (5) with wide color palettes (6). Color is tuned by playing with the nanoholes sizes and arrangement. However, by construction, such a structure is totally nontransparent at non-filtered wavelengths. Another approach is to exploit resonances in nanoparticles or in nanoantennas (7). The reflectance spectrum of a decorated substrate can be controlled by patch nanoantenna arrays or nanostructures coupled to a plasmonic film $(8,9)$, in random (10) or ordered arrangement for high spatial resolution printing (11-15). The resulting colors of the substrate are controlled by the dimensions and the shape of the nanostructures, involving LSPR or cavity resonances. Here again, the metallic parts make the optical transmittance too low for the HUD application. Using LSPR alone, arrays of metallic nanostructures provide filtering in transmission $(16)$ or in reflection $(17,18)$, with tuning induced 
either by the NP shape and size or, in dense arrays, by the LSPR coupling. Numerous implementations have also been proposed, with dielectric Mie resonances (19) or with nanowires (20). In all cases, the study was focused on single wavelength properties (reflectance or filtering), without considering the transparency of the plate on the remaining visible spectrum. For a different purpose, a plate containing randomly dispersed metallic nanoparticles has been proposed as a transparent display revealing a blue image by scattering effect (21). The display presents appropriate transparency properties, allowing the observation of objects positioned behind it, but the (real) projected image results from the scattering effect, thus the driver will need to visually accommodate on the plate to be able to read the information.

In this paper, we propose a simple design strategy to obtain a HUD transparent plate based on dipolar LSPR in $30 \mathrm{~nm}$ thick silver nanostructures giving high reflectance at specific wavelengths while keeping a globally transparent mirror. A good global transparency means more specifically a high transmittance in the remaining visible spectrum and no non-specular diffraction orders nor scattering effects in order to leave unaltered the background landscape visualization. We consider an assembly of silver ellipsoidal nanodisks structured on a glass substrate, whose NP diameters determine the reflected color (up to two colors corresponding to the two incident polarizations (22)), and spatial density controls the ratio reflectance/transmittance values of the plate. We compare these properties for both polarizations and for two different spatial arrangements of the same nanodisks: the first arrangement is on a periodic square lattice while the second is defined from a correlated disorder. We successively compare structures with a high spatial density (corresponding to subwavelength period) and then structures with decreasing densities, i.e. with longer average distance between NPs, which are 
likely to present non-specular diffraction orders in visible range. Finally, we show a qualitative demonstration of an optimized plate for HUD application.

We have fabricated silver nanorods arrays with ellipsoidal cross-section of similar diameters, arranged either in a square lattice ("Sq" arrays) or in a correlated disorder ("Rd" arrays) sharing with the "Sq" arrays similar average distances between next neighbors (Fig.1(a)). The correlated disorder arrangement is determined by using centroidal Voronoi tessalation algorithm applied on an initially random arrangement (cf Supporting information): with a rule minimizing the distance variations between adjacent NPs around a given average value, NPs are regularly positioned and sufficiently spaced so that possible LSPR coupling between adjacent NPs is homogeneous and very low. Typically the standard deviation with respect to the mean distance between adjacent NPs is of $\pm 8 \%$. Additionally, the angles between the horizontal axis and the first neighbors show no preferential direction (cf Supporting information). NPs are fabricated by electron beam lithography followed by lift-off of a $30 \mathrm{~nm}$ thick Ag layer. Thanks to the low dispersion of the mean distance between adjacent NPs, we were able to limit size variations during lithography. Each sample is then covered by a $\sim 200 \mathrm{~nm}$ thick PMMA layer to protect silver NPs from aging. This PMMA cover appears as sufficient in the present work since the samples are still available with the same properties after more than one year and with no particular precautionary measures. For safe comparison, each series of pairs of arrangements are fabricated on one same sample (fused silica glass wafer on each case):

Sample $\mathrm{N}^{\circ} 1$ (Fig.1(b)) was made to measure resonances ranging from 450 to $610 \mathrm{~nm}$ by varying the NP diameter and to compare the response of both square and correlated disordered lattices with an average sub-wavelength distance between NP of $200 \mathrm{~nm}$ for "Sq" and $216 \mathrm{~nm}$ for "Rd" arrays. The distance was selected to obtain similar density between both arrangements. 
Sample $\mathrm{N}^{\circ} 2$ (Fig.1(c)) was made to measure the effect of density on reflectance and transmittance. It contains several pairs of arrangements targeting resonances near $600 \mathrm{~nm}$ and has been fabricated with average distances from 200 to $355 \mathrm{~nm}$. The diameters and the average distances are indicated on each array.

The diameters indicated in Fig.1(b) and Fig.1(c) are obtained by averaging the diameters of multiple NP with a precision lower than $\pm 3 \mathrm{~nm}$ on three scanning electron microscope images taken at different positions. Each array is "L" shaped, with longer borders equal to $1200 \mu \mathrm{m}$ to be able to distinguish the orientation of the NP section ellipses and to take into account the reflection on the sample's backside during measurement. For $\mathrm{N}^{\circ} 1$ and $\mathrm{N}^{\circ} 2$ samples, the ellipses have their larger diameter parallel to the horizontal bar of the " $L$ " when the "L" is right side up.

(a)

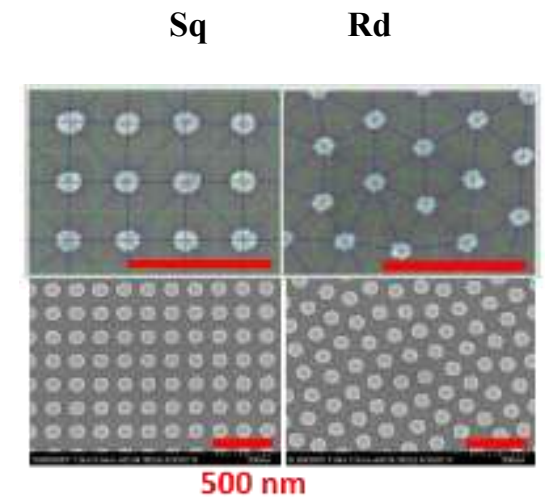

(b)

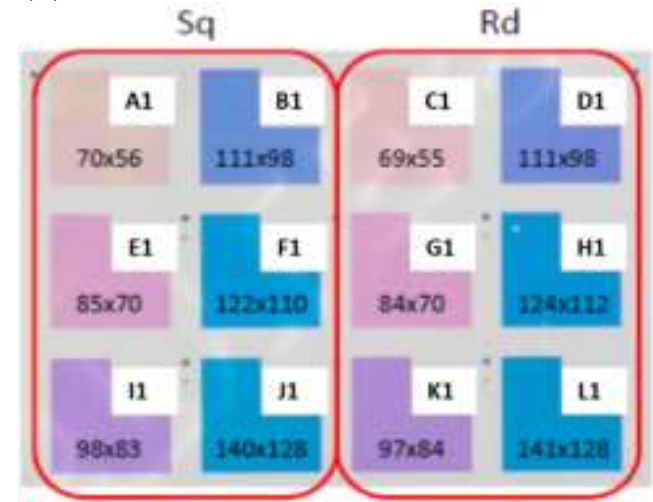

(c)

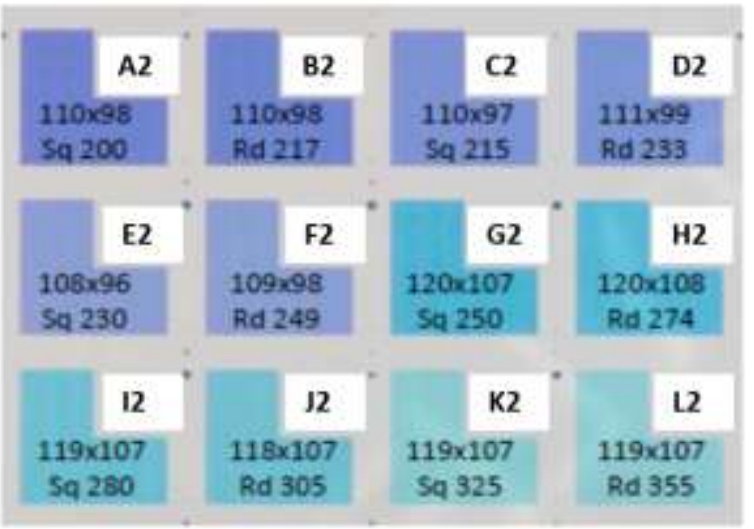


Figure 1. Fabricated samples: (a) SEM images of the square lattice (left) and correlated disorder (right) NPs arrangements. (b) Backlit photography of sample $\mathrm{N}^{\circ} 1$ with pairs of "Sq" and "Rd" arrays with 200nm (Sq) and 216nm (Rd) average interparticle distances and different ellipses diameters (formatted as “a x b" given in nm). (c) Backlit photography of sample $\mathrm{N}^{\circ} 2$ with pairs of "Sq" and "Rd" arrays with the same ellipses diameters (format "a x b" in $\mathrm{nm}$ ) and increasing average interparticles distances (in $\mathrm{nm}$ ).

Reflectance and transmittance spectra on the visible range are presented in Fig. 2(a) for two arrangement pairs of sample $\mathrm{N}^{\circ} 1$. For all measurements presented in the paper the incidence plane is orthogonal to the smaller diameter of the ellipses. The measurement is performed with a Xenon lamp followed by a monochromator. The collimated light is polarized and then focused on the sample surface with $45^{\circ}$ incident angle with an objective and the reflected beam is collected at the specular angle $\left(45^{\circ}\right)$. Transmittance is measured with a separated scan by positioning the detector behind the sample and by keeping the same angle of incidence. The pairs in Fig. 2(a) were selected because they present the same ellipse diameter (110-112 nm) in the direction of the incident electric field, for the $\mathrm{S}$ and the $\mathrm{P}$ polarizations respectively, and finally provide similar resonance wavelengths: the LSPR resonance has the widest peak near $600 \mathrm{~nm} . \mathrm{P}$ polarized incident light leads to a lower reflected intensity than S polarized light due to the partial excitation of the LSPR (projection of the electric field on the NP ellipse resonant axis). This shows that at sub-wavelength spacing the type of NPs arrangement has negligible effect on the measured spectra. The main differences between the different curves occur at shorter wavelengths (near $490 \mathrm{~nm}$ for $\mathrm{S}$ polarization reflectance, and near $410 \mathrm{~nm}$ for "Sq" arrays reflectance) and will be discussed hereafter. 
Similar measurements have been performed for both polarizations on all the arrays of sample $\mathrm{N}^{\circ}$ 1. Fig.2(b) shows the reflectance maxima of LSPR intensities versus the LSPR wavelengths. Here again, for each polarization, the sample's properties appear to depend only on the LSPR wavelength and to be insensitive to the arrangement. Besides, for the largest ellipse sections, the LSPR wavelength is slightly higher in the case of the "Sq" lattice: this is attributed to the coupling between adjacent NPs which are nearer in the "Sq" array $(200 \mathrm{~nm})$ than in the "Rd" array $(216 \mathrm{~nm})$. Indeed this coupling is no more negligible when the ellipses have diameters higher than their inter-distance. With such dense arrays (subwavelength average distance) the reflectance peak at $45^{\circ}$ reaches values from $40 \%$ at $400 \mathrm{~nm}$ up to $95 \%$ at $620 \mathrm{~nm}$, which has to be compared with the lower reflectivity of uncoated glass (lower than 10\%). The drop of the reflectance peak at lower wavelengths corresponds to the plasmonic response of silver which progressively decreases down to the interband transition threshold of silver in the near UV.

The behaviour of the periodic arrays can be modelled accurately with 3D full wave calculations using for instance the Finite Elements Method (FEM) (23) or the Finite Difference Time Domain (FDTD) method. These arrays have been finely optimized in a previous work using Finite Elements (24). Fig.2(c)-(d) shows the reflectance and transmittance spectra calculated by FEM for all the "Sq" arrays in sample $\mathrm{N}^{\circ} 1$ for both polarizations. Notice that all the optical indices used in the simulations have been obtained by ellipsometric measurements made on dedicated samples.

When comparing the simulations in Fig.2(c)-(d) to the measurements shown in Fig.2(a), it can be seen that, by taking into account the uncertainty of some fabrication steps, the position of all the peaks are in good agreement. The remaining discrepancies, with a $\sim 30 \mathrm{~nm}$ shift of the peaks between the modelling and measurements, can be attributed to the thickness mismatch and to the 
shape difference between the model (perfectly flat top for the disks) and the experiment (rounded top).
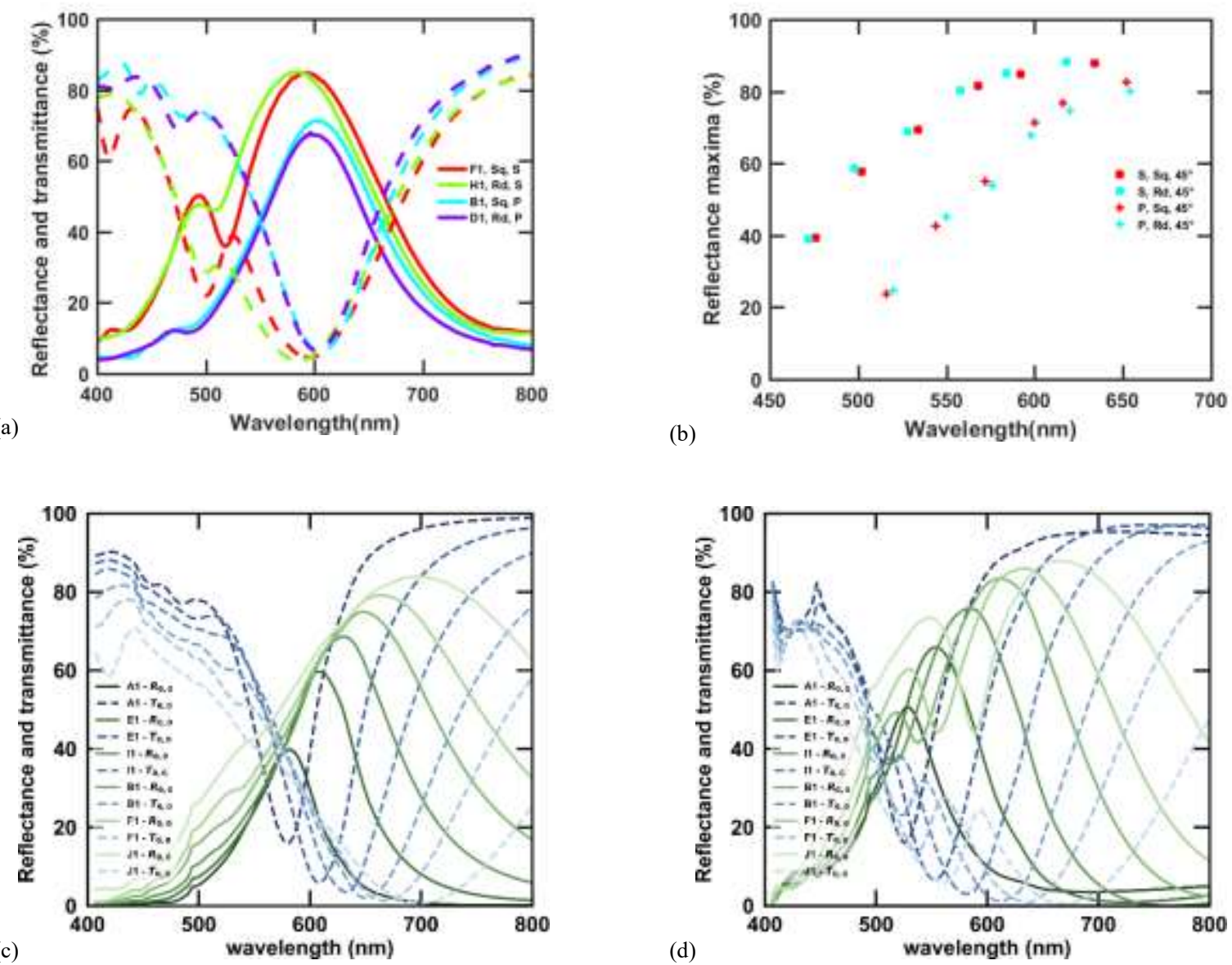

Figure 2. Reflectance (solid lines) and transmittance (dashed lines) measurements of sample $\mathrm{N}^{\circ} 1$ arrays: (a) spectra of "Sq" and "Rd" arrays with a similar NP resonant axis of $111 \pm 1 \mathrm{~nm}$, for polarizations $\mathrm{P}$ and $\mathrm{S}$ (arrays $\mathrm{B}, \mathrm{D}, \mathrm{F}$ and $\mathrm{H}$ ). The incident angle is $45^{\circ}$. (b) Reflectance maxima of LSPR intensity reported versus the resonance wavelength obtained on all the arrays, for $45^{\circ}$ incident angle and each polarization. FEM simulations for P (c) and S (d) polarization of all the "Sq" arrays of sample $\mathrm{N}^{\circ} 1$ shown in Fig.1(b). 
Measurements and simulations show that the increase in diameter clearly redshifts and broadens the reflection peak. The other important characteristic studied in this paper is the effect of NP density and arrangement on the compromise between the reflectance peak at LSPR and the averaged transmittance in the visible spectrum. Sample $\mathrm{N}^{\circ} 2$ was made to test the effect of density. It contains both "Sq" and "Rd" arrangements, and the NP diameters have been selected to present a LSPR at around $600 \mathrm{~nm}$.

Fig. 3 shows, for each of the 12 arrays, the reflectance maxima in S polarization and the averaged transmittance (for both polarizations and on the whole visible spectrum) versus the array density. Here again, both "Rd" and "Sq" arrangements give similar optical responses. For densities higher than $16 \mathrm{NPs} / \mu \mathrm{m}^{2}$, the reflectance maxima are hardly improved, whereas the averaged transmittance steadily decreases with density. With a reflectance of $70 \%$ and an averaged transmittance of $60 \%$, the $16 \mathrm{NPs} / \mu \mathrm{m}^{2}$ density appears as a good compromise for a plate dedicated to HUD application.

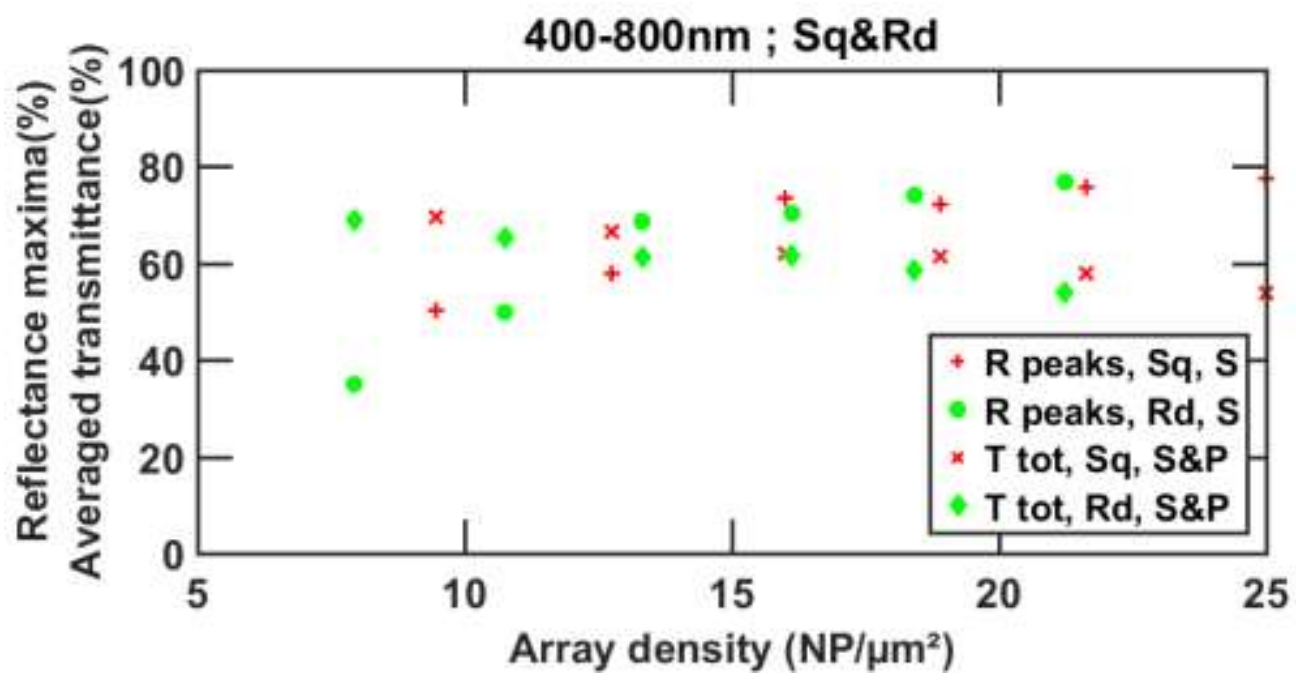

Figure 3. Reflectance maxima in $\mathrm{S}$ polarization and averaged transmittance (for both polarizations on the whole visible spectrum) versus the array density, for the 12 arrays from sample $\mathrm{N}^{\circ} 2$. 
The complete reflectance spectra of the 12 arrays in sample $\mathrm{N}^{\circ} 2$ are shown in Fig.4 for $\mathrm{S}$ polarization. For better readability, the curves are vertically shifted with a step proportional to the average distance between the NPs. In insert are shown the unshifted curves in order to easily visualize the relative intensities. Due to different lithography calibrations of the three denser arrays, the main resonance wavelength of the "Rd" arrays (represented by the full circles in Fig.4) is slightly blue-shifted with respect to the three others. This has negligible impact on the following analysis.

It can be noted that like in Fig.3, in Fig.4 the reflectance maxima decreases with the spatial density (or with increasing average distance), for both "Rd" and "Sq" arrangements. But in addition to this reflectance drop, the "Sq" arrays series is strongly disturbed by additional small drops and peaks: the one seems to red-shift with decreasing arrays density and the other is almost independent of the array density. The latter is visible on both "Sq" and "Rd" arrangements near $490 \mathrm{~nm}$ (it is also visible in Fig.2(a) of sample $\mathrm{N}^{\circ} 1$ ). In Fig.4, for comparison between "Sq" and "Rd" arrangements, the peak positions for the "Rd" arrays are marked by empty squares. 
+ Grazing diffraction modes

- Dipolar resonances

- Quadrupolar resonances
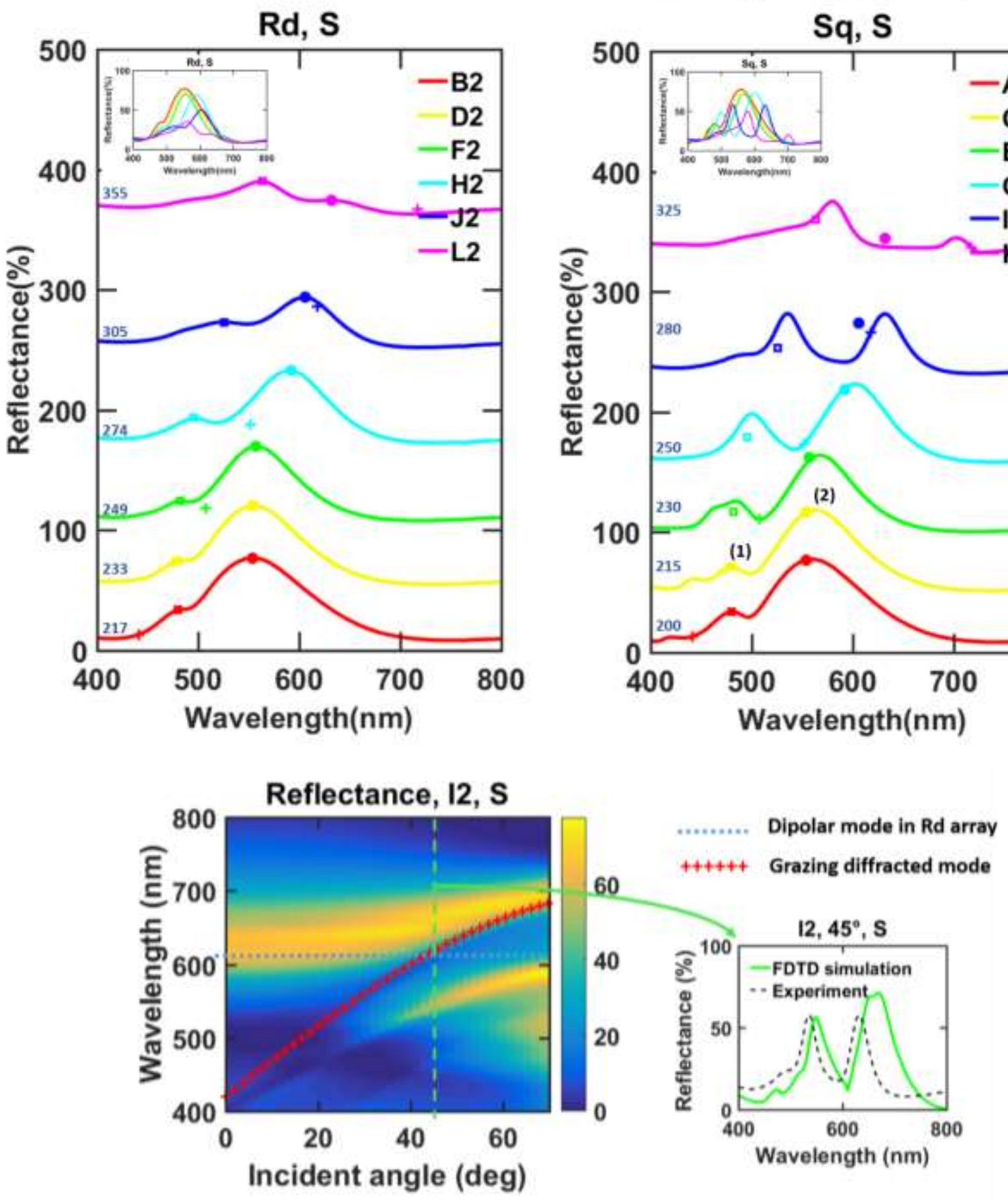
Figure 4. Top: reflectance measured on the 12 arrays of sample $\mathrm{N}^{\circ} 2$, with $\mathrm{S}$ polarized illumination at $45^{\circ}$ incident angle, vertically shifted proportionally to the NPs average distance for better readability (in insert, the same curves without the vertical shift to highlight the relative intensities): filled circles and empty squares indicate the wavelengths of respectively the dipolar and quadrupolar resonances measured on "Rd" arrays and reported on "Sq" arrays for comparison; crosses are the calculated grazing diffracted modes (Equ. 1); blue numbers are the average distance between NPs (nm). In insert above: FDTD simulated perpendicular component of the electric field in the NPs of array C2 (top view) at two resonant wavelengths: (1) quadrupolar and (2) dipolar resonances. Bottom: reflectance curves of array I2, calculated by FDTD, versus the incident angle for $\mathrm{S}$ polarization, and cross view of the $45^{\circ}$ response superimposed on the experimental data. The blue dotted line indicates dipolar mode extracted from $\mathrm{J} 2$ array measurement at $45^{\circ}$ and with $\mathrm{S}$ polarization.

This density independent peak can be attributed either to higher order plasmonic modes (like quadrupolar mode) or to an additional Fabry-Perot mode guided in the PMMA cover layer above the silver NP array. To understand the origin of this peak we made FDTD simulations with a commercial software (FDTD solutions, Lumerical Inc.). The simulations have been performed in the case of the periodic array $\mathrm{C} 2$ of sample $\mathrm{N}^{\circ} 2$ by taking into account the $200 \mathrm{~nm}$ PMMA cover layer. A mesh size of $2 \mathrm{~nm}$ has been used for the simulations and silver optical index has been deduced from experimental data. Maps of the electric field in the plane of the NPs obtained with an incident angle of $45^{\circ}$ are inserted in Fig.4 (top) for two remarkable reflectance peaks of the square lattice array $\mathrm{C} 2$. These maps confirm the excitation of a quadrupolar mode below $500 \mathrm{~nm}$. The simulated and experimental peak wavelengths are in good agreement, differences come from the uncertainty on silver thickness during the fabrication process and the shape difference 
between the model (perfectly flat top for the disks) and the experiment (rounded top). Besides, the hypothetic additional Fabry-Perot mode guided in the PMMA cover layer is expected to induce a reflectance peak dependent on the incident angle. Thus as an additional check, in Fig.5 we measured the reflectance for both polarizations at different angles on an array with "Rd" arrangement and a density of $16 \mathrm{NPs} / \mu \mathrm{m}^{2}$. The secondary reflectance peak occurs at $490 \mathrm{~nm}$ regardless of the angle and the polarization. It also appears to be more efficiently excited when the angle increases. That effect is more accentuated in the case of $\mathrm{S}$ polarization. These behaviours are signatures of multipolar plasmonic resonances and exclude the presence of FabryPerot guided mode. It can also be noted that the P polarized signal appears slightly dependent on the incident angle (Fig.5, right), because of angle dependent projections of the electric field.
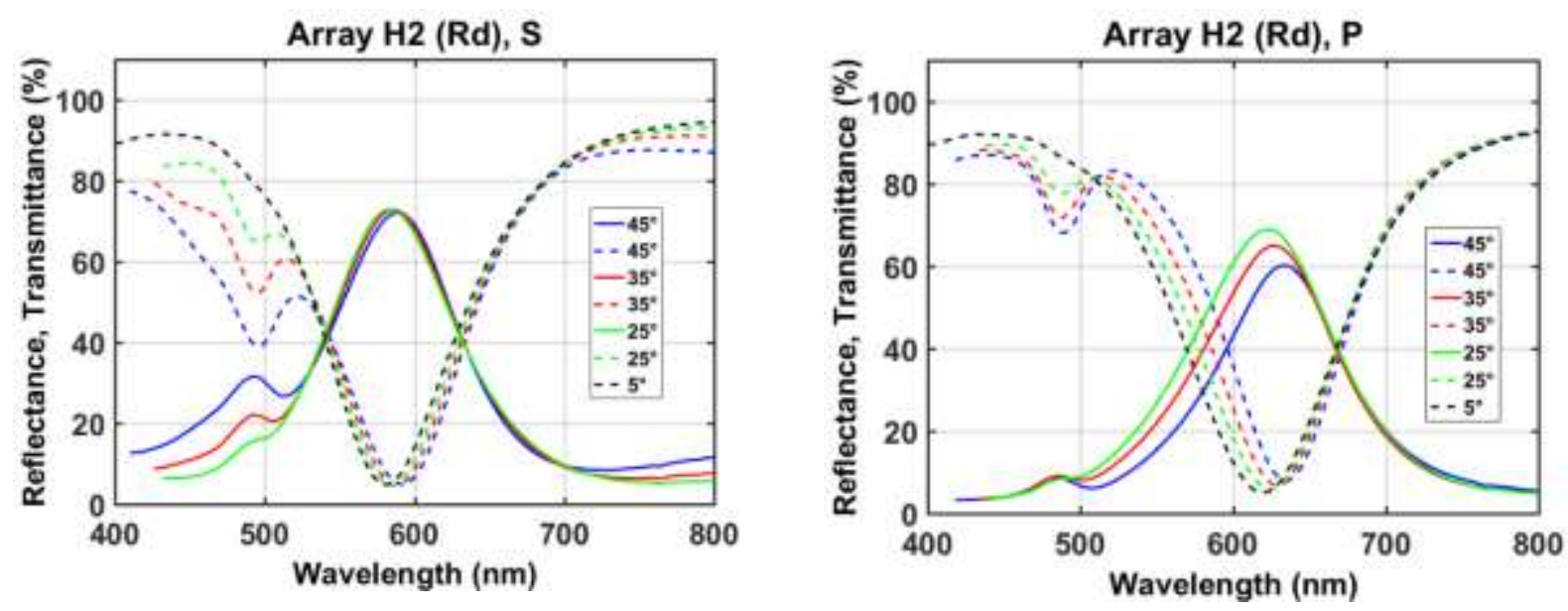

Figure 5. Reflectance and transmittance measurements on sample $\mathrm{N}^{\circ} 3$ at different angles and for both polarizations. This sample has a large area $\left(1 \mathrm{~cm}^{2}\right)$ with the design of array H2 (Rd arrangement, $120 \times 108 \mathrm{~nm}$ diameters, $271 \mathrm{~nm}$ average distance), the spectra were averaged on multiple locations.

In addition, the array density dependent drop is not present in the response of " $\mathrm{Rd}$ " arrays and hardly in the case of "Sq" arrays in P polarization (Fig.4 and Fig.6). This can be attributed to the diffraction phenomena. Indeed, lattice surface modes (LSM) are induced by the coupling of 
diffractive Rayleigh anomalies and LSPR, and are likely to modify the specular reflectance. Such effects have been observed in several systems involving periodic plasmonic NP arrays (25-29). The position of the relevant Rayleigh anomalies can be deduced from the 1D grating formula for transmitted orders: $n_{\text {sub }} \sin \theta_{m}^{t}=n_{\text {sup }} \sin \theta_{i}+m \lambda_{0} / a$, where $\theta_{i}$ is the incident angle $\left(45^{\circ}\right.$ here), $\theta_{m}^{t}$ the angle of the $m^{\text {th }}$ transmitted diffraction order, $\lambda_{0}$ the wavelength of the incident field in air, $n_{\text {sup }}$ the superstrate index, $n_{\text {sub }}$ the substrate index and $a$ the period of the grating. The first grazing transmitted diffraction order rises as $\theta_{1}^{t}$ equals $90^{\circ}$ and thus the concerned wavelengths of first order are:

$$
\lambda_{0}=a / m\left(n_{\text {sub }}+\frac{n_{\text {sup }} \sqrt{2}}{2}\right) \quad \text { (Equ. 1) }
$$

In our case, for a period of $200 \mathrm{~nm}$ and an incident angle of $45^{\circ}, \lambda_{0}=446 \mathrm{~nm}$ (Red Cross in Fig. 4 (top) and Fig.6).
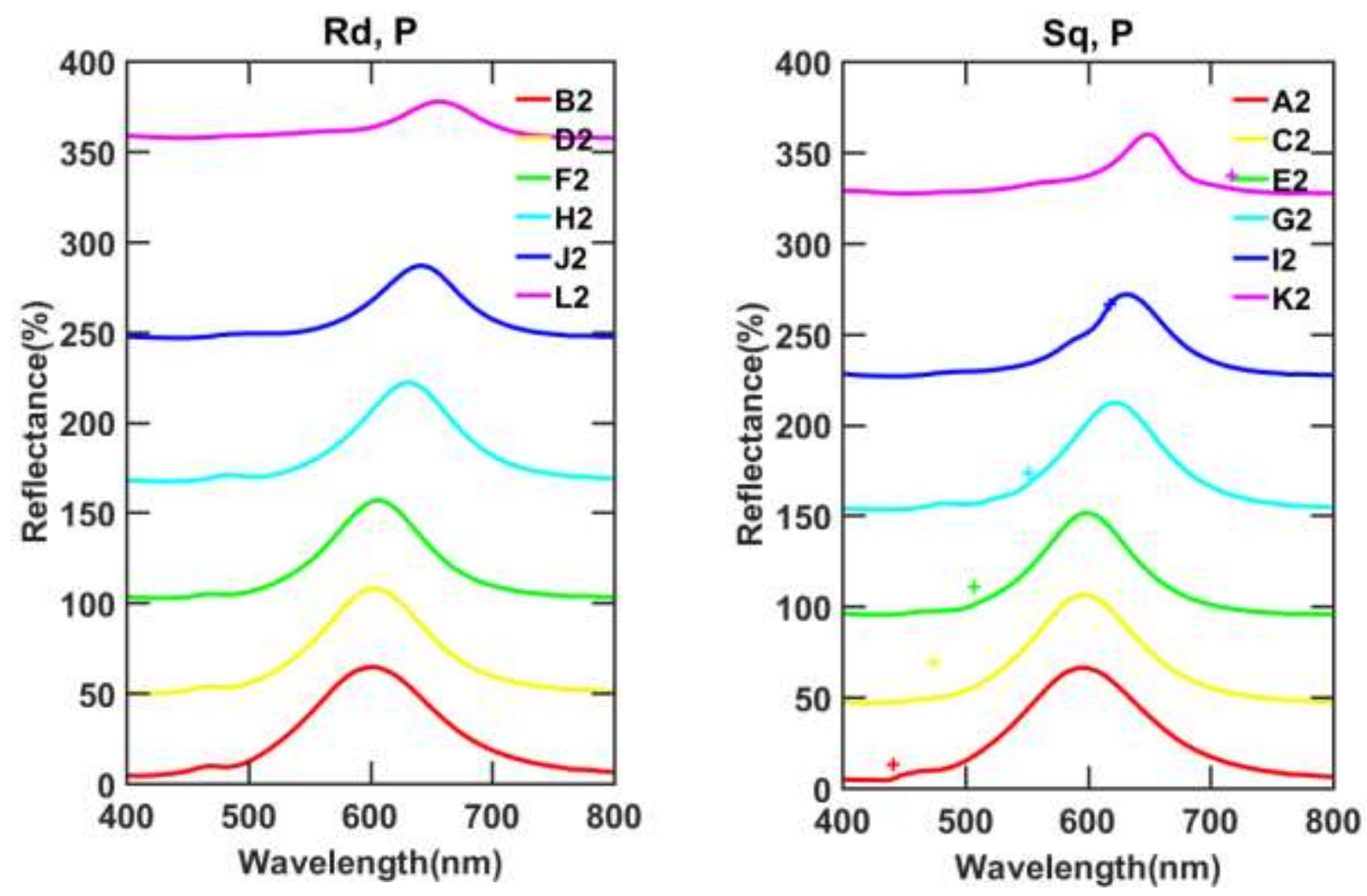
Figure 6. Reflectance measured on the 12 arrays of sample $\mathrm{N}^{\circ} 2$, with $45^{\circ}$ incident angle and $\mathrm{P}$ polarization. Crosses correspond to the calculated grazing first transmitted diffraction order.

In the case of periodic NPs arrays, narrow Rayleigh anomalies may couple with wide LSPR. Depending on the relative spectral positions of the both resonances (30), the interaction can be out-of-phase or in-phase and results in a weak response (like in the case of array A2, at $\lambda=$ $410 \mathrm{~nm}$, Fig.4(top)), or deep effect (array I2, at $\lambda \sim 600 \mathrm{~nm}$, Fig.4(top and bottom)). Additionally, as recently described by Guo et al (31), LSM in plasmonic NPs array presents a strong polarization dependence: the S polarization excites transverse plasmonic resonances in the NPs along the incident plane, whereas $\mathrm{P}$ polarization excites transverse plasmonic resonances in the perpendicular plane of the incidence, with much lower interaction with the Rayleigh anomalies. Here, P polarization diffraction anomalies are well located by the simple grating formula in case of first order corresponding to $\theta=90^{\circ}$ (crosses in Fig.6), but they are very weak. On the other hand, S polarization diffraction anomalies show more interaction with the dipolar resonance: for the denser arrays, they can be identified independently of the quadrupolar and the dipolar resonances, whereas for the arrays G2, I2 and K2 clear coupling occurs with dipolar resonances only (32). Dispersion curve of the array I2, calculated by FDTD (Fig.4, bottom), shows the coupling between the diffractive Rayleigh anomaly (red crosses) and the plasmonic dipolar resonance (identified in the corresponding "Rd" array J2). At $45^{\circ}$ incident angle, the two resonances coupling results in the split in two main peaks. The simulated and experimental responses are in good agreement, taking into account the manufacturing uncertainties, and the only $2 \mathrm{~nm}$ spectral resolution of the experimental setup. Smaller wavelength steps are required to fully resolve the very narrow LSM. Besides, the range of array periods considered in the samples does not allow the observation of the dip and sharp peak corresponding to in-phase interaction of 
separated resonances (30), which should occur at larger periods. All these behaviours can be also observed, with more accentuated profiles, in transmittance measurement (Supporting information).

Now, the present measurement results are discussed according to the targeted application of this work. S polarization provides better trade-off reflectance/transmittance while P polarization results in slightly narrower reflectance peak at $45^{\circ}$ (because of weaker quadrupolar resonance) and disordered arrangement allows lower array density and higher plate transmittance thanks to limited diffraction effects. In a HUD system, the plate transparency requires the two following properties: not only high optical transmittance but also negligible scattering effects and clear reflected picture and landscape view. Disordered arrays are likely to induce scattering of the light, especially at shorter wavelengths. In the present work, correlated disorder was introduced to avoid dispersive resonance coupling between plasmonic NPs, and is at the same time a way to limit scattering effect as described in (33). It also avoids the broadening of the reflectance peak due to inhomogeneous long range interaction between NPs in fully random arrangement as shown in (34). Sample $\mathrm{N}^{\circ} 3$ was made to perform macroscopic observation of correlated disorder NPs arrangement on a large area $\left(1 \mathrm{~cm}^{2}\right)$ and with the characteristic of the array $\mathrm{H} 2$ of sample $\mathrm{N}^{\circ} 2(120 \times 108 \pm 4 \mathrm{~nm}$ diameters, $271 \pm 23 \mathrm{~nm}$ average distance). As shown in Fig.5, S and P polarizations reflectance peaks are positioned at $590 \mathrm{~nm}$ with maximum of respectively $70 \%$ and $60 \%$ in intensity. Plate transparency can be qualitatively assessed in Fig.7(a) which shows the same landscape image without windshield and through the fabricated plate. In the second case the image remains clear and appears slightly bluish. HUD like experiment was performed with realistic amber-colored display reflection corresponding to resonance around $590 \mathrm{~nm}$ (Fig.7(b)). The "color diagram" of the reflected picture is very similar to the original one (Fig.7(c)). 
(a)

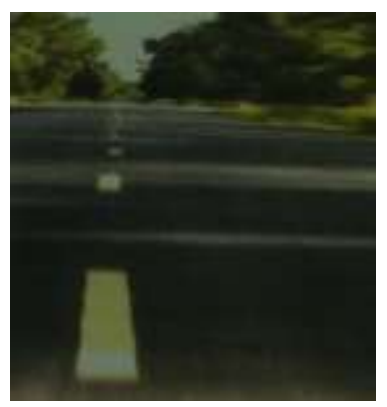

(b)

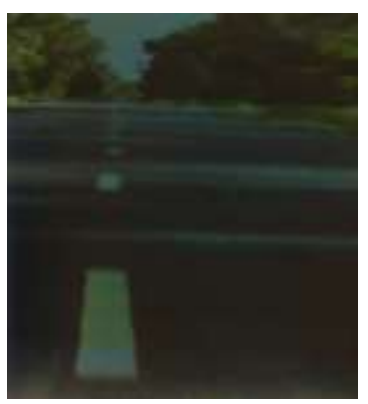

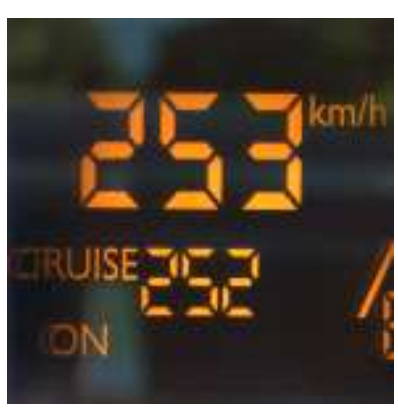

(c)

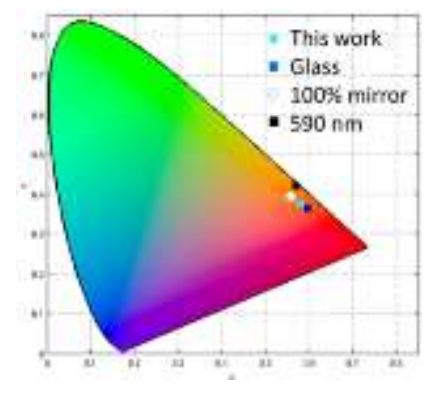

Figure 7. (a) Road image without (left) and through (right) the plasmonic plate. (b) HUD like experiment with the fabricated plasmonic plate, and (c) color description on color gamut (clear blue square), to be compared with reflected image on glass only (dark blue square), perfect mirror (white circle) and with '590 nm' color (black square).

To conclude, we have shown that subwavelength square lattice arrays present similar optical properties to the correlated disordered arrays with the same spatial density, regardless of the resonant wavelength and the polarization (P or S). By decreasing the spatial density (longer average distance between NPs), diffraction orders and lattice surface modes induce strong perturbation in the specular reflectance spectrum of the "Sq" (regular) lattice. However, the impact of this long distance effect is considerably reduced in $\mathrm{P}$ polarization due to lower efficiency of surface lattice resonance excitation. Additionally, these grazing diffraction peaks can be strongly attenuated in the case of the correlated disordered arrangement. Besides, whereas S polarization provides better trade-off reflectance/transmittance, $\mathrm{P}$ polarization offers narrower peaks thanks to lower quadrupolar mode excitation at $45^{\circ}$. Lastly, we checked that a wide range of reflectance/averaged transmittance ratio can be obtained by using spatial arrangements based on correlated disorder of the NPs, simultaneously avoiding diffraction (in the visible range) and scattering (for image and transparency quality) effects, and keeping resonance peaks widths similar to the "Sq" lattice case, and thus narrower than in fully random arrangements. A large 
area sample has been fabricated and successfully tested in a representative lab visualization system. These promising results pave the way for HUD systems and could find some innovative applications in the transport industry such as in the automotive or aeronautic sectors for displays, human-machine interfaces, and sensors.

\section{REFERENCES}

(1) Fehrembach, A.-L.; Popov, E.; Tayeb G.; Maystre, D. Narrow-band filtering with whispering modes in gratings made of fibers. Opt. Expr. 2007, 15, 15734 -15740.

(2) Genet, C.; Ebbesen, T.W. Light in tiny Holes. Nature 2007, 445, 39-46.

(3) Lee, H.-S.; Yoon, Y.-T.; Lee,S.-S.; Kim, S.-H.; Lee, K.-D. Color filter based on a subwavelength patterned metal grating. Opt. Expr. 2007, 15, 15457-15463.

(4) Yokogawa, S.; Burgos, S. P.; Atwater, H. A. Plasmonic color filters for CMOS image sensor applications. Nano Lett. 2012, 12, 4349-4354.

(5) Cheng, F.; Gao, J.; Stan, L.; Rosenmann, D.; Czaplewski, D.; Yang, X. Aluminum plasmonic metamaterials for structural color printing. Opt. Expr. 2015, 23, 14552-14560.

(6) Li, Z.; Clark, A. W.; Cooper, J. M. Dual color plasmonic pixels create a polarization controlled nano color palette. ACS Nano 2016, 10, 492-498.

(7) Kristensen, A; Yang, J.K.W.; Bozhevolnyi, S.I.; Link, S.; Nordlander, P.; Halas, N.J.; Mortensen N.A. Plasmonic colour generation. Nature Rev. Mat. 2016, 2, 16088.

(8) Maurer, T.; Adam, P.-M.; Lévêque, G. Coupling between plasmonic films and nanostructures: from basics to applications. Nanophotonics 2015, 4, 363-382. 
(9) Zhu, X.; Vannahme, C.; Højlund-Nielsen, E.; Mortensen, N.A; Kristensen, A. Plasmonic colour laser printing. Nature Nanotech. 2016, 11, 325-329.

(10) Moreau, A.; Ciraci, C.; Mock, J.J.; Hill, R.T.; Wang, Q; Wiley, B.J.; Chilkoti, A.; Smith, D.R. Controlled-reflectance surfaces with film-coupled colloidal nanoantennas. Nature 2012, 492, 86-90.

(11) Kumar, K.; Duan, H.; Hedge, R.S.; Koh, S.C.W.; Wei, J.N.; Yang, J.K.W. Printing colour at the optical diffraction limit. Nature Nanotech. 2012, 7, 557-561.

(12) Tan, S.J.; Zhang, L.; Zhu, D. ; Goh, X.M. ; Wang, Y.M.; Kumar, K.; Qiu, C.-W.; Yang, J.K.W. Plasmonic color palettes for photorealistic printing with aluminum nanostructures. Nano Lett. 2014, 14, 4023-4029.

(13) Roberts, A.S.; Pors, A.; Albrektsen, O.; Bozhevolnyi, S.I. Subwavelength Plasmonic Color Printing Protected for Ambient Use. Nano Lett. 2014, 14, 783-787.

(14) Miyata, M.; Hatada, H. Takahara, J. Full-Color Subwavelength Printing with GapPlasmonic Optical Antennas. Nano Lett. 2016, 16 (5), 3166-3172.

(15) Li, Z.; Wang, W.; Rosenmann, D.; Czaplewski, D.A.; Yang, X.; Gao, I. All-metal structural color printing based on aluminum plasmonic metasurfaces. Opt. Expr. 2016, 24(18), 20472-20480.

(16) Ellenbogen, T.; Seo, K.; Crozier, K. B. Chromatic plasmonic polarizers for active visible color filtering and polarimetry. Nano Lett. 2012, 12, 1026-1031. 
(17) Si, G. ; Zhao, Y. ; Lv, J. ; Lu, M. ; Wang, F. ; Liu, H. ; Xiang, N. ; Huang, T.J. ; Danner, A.J. ; Teng, J. ; Liu, Y.J. Reflective plasmonic color filters based on lithographically patterned silver nanorod arrays. Nanoscale 2013, 5, 6243-6248.

(18) Ng, R.J.H; Goh, X.M.; Yang, J.K.W. All-metal nanostructured substrates as subtractive color reflectors with near-perfect absorptance. Opt. Expr. 2015, 23(25), 32597-32605.

(19) Sun, S.; Zhou, Z.; Zhang, C.; Gao, Y.; Duan, Z. ; Xiao, S. ; Song, Q. All-Dielectric FullColor Printing with TiO2 Metasurfaces. ACS Nano 2017, 11(5), 4445-4452.

(20) Park, H.; Dan, Y.; Seo, K.; Yu, Y.J.; Duane, P.K.; Wober, M.; Crozier, K.B. Filter-free image sensor pixels comprising silicon nanowires with selective color absorption. Nano Lett. 2014, 14(4), 1804-1809.

(21) Hsu, C.W.; Zhen, B.; Qiu, W.; Shapira, O.; Delacy, B.G.; Joannopoulos, J.D.; Soljacic, M. Transparent displays enabled by resonant nanoparticle scattering. Nature Comm. 2014, 4152.

(22) Goh, X. M.; Zheng, Y.; Tan, S.J.; Zhang, L.; Kumar, K.; Qiu, C.-W.; Yang, J.K.W. Threedimensional plasmonic stereoscopic prints in full colour, Nature Comm. 2014, 5, 5361.

(23) Demésy, G; Zolla, F.; Nicolet, A ; Commandré, M. All-purpose finite element formulation for arbitrarily shaped crossed-gratings embedded in a multilayered stack. JOSA A 2010, 27(4), 878-889.

(24) Brûlé, Y.; Demésy, G.; Fehrembach, A.-L.; Gralak, B.; Popov, E.; Tayeb, G.; Grangier, M.; Barat, D.; Gogol, P.; Dagens, B. Design of metallic nanoparticles gratings for filtering properties in the visible spectrum. Appl. Opt. 2015, 54, 010359. 
(25) Carron, K. T.; Fluhr, W. ; Meier, M.; Wokaun, A.; Lehmann, H.W. Resonances of twodimensional particle gratings in surface-enhanced Raman scattering. J. Opt. Soc. Am. B 1986, 3, 430-440.

(26) Garcia de Abajo, F. J. Colloquium: Light scattering by particle and hole arrays. Rev. Mod. Phys. 2007, 79, 1267.

(27) Zou, S.; Shatz, G.C. Narrow plasmonic/photonic extinction and scattering line shapes for one and two dimensional silver nanoparticle arrays. J. of Chem. Phys. 2004, 121(24), 1260612612.

(28) Kravets, V.G.; Schedin, F.; Grigorenko, A.N. Extremely Narrow Plasmon Resonances Based on Diffraction Coupling of Localized Plasmons in Arrays of Metallic Nanoparticles. Phys. Rev. Lett. 2008, 101, 087403.

(29) Auguié, B.; Barnes, W.L. Collective Resonances in Gold Nanoparticle Arrays. Phys. Rev. Lett. 2008, 101, 143902.

(30) Teperik, T.V.; Degiron, A. Design strategies to tailor the narrow plasmon-photonic resonances in arrays of metallic nanoparticles. Phys. Rev. B 2012, 86, 245425.

(31) Guo, R. ; Hakala, T. K. ; Törmä, P. Geometry dependence of surface lattice resonances in plasmonic nanoparticle arrays. Phys. Rev. B 2017, 95, 155423.

(32) Teperik, T.V.; Degiron, A. Superradiant Optical Emitters Coupled to an Array of Nanosize Metallic Antennas. Phys. Rev. Lett. 2012, 108, 147401. 
(33) Conley, G.M.; Burresi, M.; Pratesi, F.; Vynck, K.; Wiersma, D.S. Light Transport and Localization in Two-Dimensional Correlated Disorder. Phys. Rev. Lett. 2014, 112, 143901.

(34) Nishijima, Y.; Lorenzo Rosa, L.; Juodkazis, S. Surface plasmon resonances in periodic and random patterns of gold nano-disks for broadband light harvesting. Opt. Expr. 2012, 20, $11466-11477$.

\section{ASSOCIATED CONTENT}

Supporting Information: Correlated disorder arrangement; Transmittance measurements on sample $\mathrm{N}^{\circ} 2$.

\section{AUTHOR INFORMATION}

\section{Corresponding Author}

*Beatrice Dagens, beatrice.dagens@c2n.upsaclay.fr.

\section{Author Contributions}

The manuscript was written through contributions of all authors. All authors have given approval to the final version of the manuscript.

\section{Funding Sources}

This work has been supported by the ANR funded project PLANISSIMO (ANR-12-NANO0003), by the french RENATECH network and by the PSA Groupe - Université Paris-Sud Optoelectronics and Photonics research chair. 


\section{ABBREVIATIONS}

NP, nanoparticle; HUD, Head-up display; LSPR, localized surface plasmon resonance; EOT, extraordinary optical transmission; PMMA, polymethacrylatemethyl; SEM, scanning electron

microscope; FEM, Finite Elements Method; FDTD, Finite Difference Time Domain; LSM, lattice surface modes. 


\section{For Table of Contents Use Only}

\section{Correlated disordered plasmonic nanostructures}

\section{arrays for augmented reality}

${ }^{1}$ Herve Bertin, ${ }^{2}$ Yoann Brûlé, ${ }^{1}$ Giovanni Magno, ${ }^{1}$ Thomas Lopez, ${ }^{1}$ Philippe Gogol, ${ }^{3}$ Laetitia Pradere, ${ }^{2}$ Boris Gralak, ${ }^{3}$ David Barat, ${ }^{2}$ Guillaume Demésy, ${ }^{1}$ Beatrice Dagens*

${ }^{1}$ Centre de Nanosciences et de Nanotechnologies, CNRS, Université Paris-Sud, Université ParisSaclay, C2N-Orsay, 91405 Orsay Cedex, France

${ }^{2}$ Aix Marseille Univ, CNRS, Centrale Marseille, Institut Fresnel, Marseille, France ${ }^{3}$ PSA groupe, Direction Scientifique, Centre technique de Vélizy, route de Gisy, VélizyVillacoublay, F-78140, France
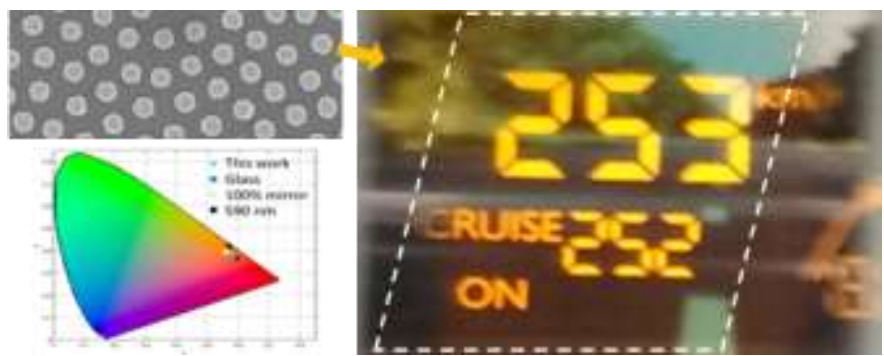

This table of content graphic illustrates the fact that the fabricated silver nanoparticle array with correlated disorder arrangement fabricated on glass induces high reflectance at orange color, preserving the reflected image readability and the landscape image quality.

Thanks to a systematic comparison between periodic and correlated disorder silver NP arrays on glass, this work successfully evaluates the use of plasmonics in transparent optical elements for visualization applications, which is a new and disruptive usage of plasmonics. 